Oprya Ye. V., Pustovoyt M. M., Biesieda Ya. V., Kozishkurt Ye. V. Pecularities of mental pathology at psychiatric patient with the comorbid somatic disorders. Journal of Education, Health and Sport. 2021;11(05): 348-355. eISSN 2391-8306. DOI http://dx.doi.org/10.12775/JEHS.2021.11.05.037

https://apcz.umk.pl/czasopisma/index.php/JEHS/article/view/JEHS.2021.11.05.037

https://zenodo.org/record/5660193

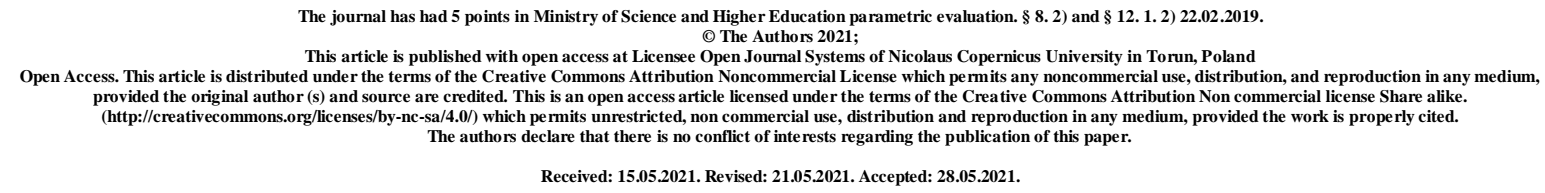

UDC 616.895.8-009.83

\title{
PECULARITIES OF MENTAL PATHOLOGY AT PSYCHIATRIC PATIENT WITH THE COMORBID SOMATIC DISORDERS
}

\author{
Ye. V. Oprya, M. M. Pustovoyt, Ya. V. Biesieda, Ye. V. Kozishkurt
}

\author{
Odessa National Medical University, Odessa, Ukraine
}

\begin{abstract}
The paper presents the results of a comprehensive clinical and psychopathological examination of patients with schizophrenia comorbid with somatic pathology. The etiological factors and preconditions for the formation of schizophrenia combined with somatic pathology, general clinical and dynamic characteristics, and features of mental disorders, severity and specificity of clinical and psychopathological manifestations of schizophrenia are analyzed. It was found that the combination of schizophrenia with somatic diseases is more "malignant" variants of the course of psychotic disorders, including an increase in the number of hospitalizations, earlier manifestation, significant increase duration and greater severity of psychopathological symptoms.

The specificity of mental disorders in patients with schizophrenia with somatic disorders was manifested by a predominance of disorders of sensation and perception in the form of
\end{abstract}


senestopathy (schizophrenia with CVD and F 20 with obesity), hyperesthesia (schizophrenia with diabetes 2) and complex hallucinations.

An important characteristic of mental disorders in patients with schizophrenia with somatic pathology is the presence of emotional disorders in the form of rigidity (in schizophrenia with CVD and obesity) and explosiveness (in schizophrenia with diabetes 2) emotional reactions, as well as depressive and anxiety (schizophrenia with CVD) and dysphoric (in schizophrenia with diabetes 2 and obesity) mood disorders. According to the frequency analysis of clinicalbiological and social factors of formation, as well as features of clinical-psychopathological structure of schizophrenia in patients of the examined groups, diagnostic clinicalpsychopathological signs (risk markers) of combination of schizophrenia with specific variants of somatic disorders are established.

The obtained results show that the combination of schizophrenia with somatic disorders is reflected in the deepening and peculiarities of psychopathological symptoms, reducing the level of social realization of patients and requires the attention of clinicians in developing treatment strategies.

Key words: comorbidity; schizophrenia; somatic pathology; clinical and psychopathological characteristics; diagnostic signs-markers.

Introduction. Comorbidity in psychiatric practice is a heterogeneous category, its varieties include general (combination of mental disorder with somatic or neurological disease), psychiatric (combination of two or more mental disorders) and mixed (mental disorder combined with mental and somatic pathology).

The most common combination for mental disorders is general comorbidity $(50 \%$ of patients), psychiatric comorbidity is registered in $42 \%$ of cases, mixed - in $8 \%$ of cases [1]. General medical comorbidity, due to its high prevalence, difficulties in diagnosis and therapy and negative medical and social consequences, is becoming increasingly important and is becoming a priority in both general practice and psychiatry, which is reflected in the strategic documents of the World Health Organization. in particular the WHO Action Plan for the Prevention and Control of Noncommunicable Diseases in the European Region, 2016-2025 [2].

It is noted that the problem of comorbidity of mental and somatic diseases includes clinical and organizational aspects. The clinical aspect has a diagnostic (overlapping symptoms, 
complications of diagnosis) and therapeutic (destabilization, drug interactions, side effects) components, which significantly and negatively affect the course and outcome of both mental and somatic disorders, adherence to therapy, quality of life patients and their relatives [1]. The organizational aspect includes the need to adapt health services to provide comprehensive care for comorbid disorders, including the creation of integrated psychiatric and somatic care, which requires appropriate structural health care reform $[1,3]$.

According to statistics for 2016, the prevalence of schizophrenia is $0.28 \%$. Globally, the prevalence of schizophrenia has increased significantly from 13.1 million in 1990 to 20.9 million in 2016 [4]. Schizophrenia is one of the most dangerous in terms of social prognosis of diseases, which is associated with a high standard of living with a disability and is one of the first in the ranking of the burden of disease [5, 6]. According to the WHO, schizophrenia is one of the 10 most common causes of loss of ability and disability. This disease is the main reason for hospitalization of patients with mental disorders and leads in the level of disability among other mental pathologies $[4,7]$.

These data suggest that schizophrenia is a complex pathology with a high hereditary risk and is increasingly recognized as a systemic (multifactorial) disorder [8]. Therefore, patients with schizophrenia face an additional burden of concomitant somatic pathology, which involves the interaction and impact of various pathological mechanisms of the disease [9].

In this case, in contrast to the causes of somatic disorders in schizophrenia, it is undeniable that the combined existence of mental and somatic pathology leads to mutual transformation of the clinical picture and significantly complicates the treatment of both somatic pathology and schizophrenia [9-11].

However, despite numerous publications on the epidemiology, consequences and etiopathogenesis of comorbid somatic disorders in schizophrenia, there is little detailed data on the pathoplastic effects of mental and somatic disorders in the available literature. In the study of the interaction of somatic and mental illness, there are two qualitatively different areas: the first is related to the study of the clinical picture and patterns of course and outcome of somatic diseases comorbid with schizophrenia; the second - with the identification of the influence of somatic pathology on the formation of clinical and psychopathological picture and the peculiarities of the actual psychopathological process combined with somatic pathology. The vast majority of the presented works are performed within the somatological direction, in which the peculiarities of the 
course and prognosis of specific variants of somatic disorders in patients with schizophrenia are studied [12].

The works are devoted to the psychopathological features of schizophrenia, which it acquires in combination with specific variants of somatic disorders are isolated and devoted to various aspects of this problem. The available work proves that the combination of schizophrenia with somatic disorders causes a certain specificity of the clinical manifestations of both pathological processes, but the question of the clinical features of schizophrenia in the case of its combination with somatic pathology still remains open today.

Aim of the work: There is an urgent need to identify clinical markers of schizophrenia, combined with specific variants of somatic disorders in order to improve the quality of their diagnosis, prevention and treatment.

\section{Material and methods}

In accordance with the purpose and objectives of the study, three main and one control group of the study were formed. The main research groups consisted of 136 patients with schizophrenia, which was combined with somatic diseases. In total, three main groups were formed.

The first main group of the study consisted of 50 patients with schizophrenia with chronic cardio-vascular disease (CVD), which at the time of the study were compensated. Patients with post-stroke and post-infarction conditions were not included in the study.

The second main group of the study presented 42 patients with schizophrenia with type 2 diabetes mellitus (DM 2) without signs of obesity $(\mathrm{BMI}<30)$.

The third main group of the study consisted of 44 patients with schizophrenia with a diagnosis of obesity (provided that the body mass index (BMI $\geq 30$ ).

The control group was formed from patients with schizophrenia without chronic somatic disorders and numbered 50 people.

The following methods were used as research tools: psychometric (Positive and Negative Syndrome Scale - PANSS), psychodiagnostic and clinical-statistical (frequency analysis by comparison groups using the procedure of sequential statistical analysis with diagnostic coefficient (DC) and measure of informativeness (MI). 


\section{Results}

According to the results of a comprehensive clinical and psychopathological examination of patients of the research groups, the etiological factors and preconditions for the formation of schizophrenia combined with somatic pathology, general clinical and dynamic characteristics, features of mental disorders, severity and specificity of clinical and psychopathological manifestations of schizophrenia are analyzed.

It was found that the combination of schizophrenia with somatic diseases is more "malignant" variants of the course of psychotic disorders, including an increase in the number of hospitalizations, earlier manifestation, significant increase duration and greater severity of psychopathological symptoms.

The following are defined as etiopathogenetic factors involved in the formation of somatic pathology in schizophrenia: in the formation of comorbidity with CVD - a history of psychoactive substance abuse, hereditary burden of addictive and CVD disorders; in the formation of comorbid obesity - hereditary burden of mental pathology (affective, neurotic and personality disorders, presented in parents); in the formation of schizophrenia, which is comorbid with diabetes mellitus 2 - hereditary burden of diabetes mellitus (without determining the type).

The specificity of mental disorders in patients with schizophrenia with somatic disorders was manifested by a predominance of disorders of sensation and perception in the form of senestopathy (schizophrenia with CVD and F 20 with obesity), hyperesthesia (schizophrenia with diabetes 2) and complex hallucinations, including, in addition to visual and auditory hallucination was added visceral hallucinations and hallucinations of the skin. An important characteristic of mental disorders in patients with schizophrenia with somatic pathology is the presence of emotional disorders in the form of rigidity (in schizophrenia with CVD and obesity) and explosiveness (in schizophrenia with diabetes 2) emotional reactions, as well as depressive and anxiety (schizophrenia with CVD) and dysphoric (in schizophrenia with diabetes 2 and obesity) mood disorders.

After conduct of PANSS interview of research group next peculiarities were revealed. The clinical and psychopathological structure of schizophrenia with CVD is characterized by the severity of hallucinatory behavior $\left(\mathrm{P}_{3}=4.17 \pm 0.19\right)$, somatic concerns $\left(\mathrm{G}_{1}=4.19 \pm 0.18\right)$, anxiety $\left(\mathrm{G}_{2}=3.74 \pm 0.16\right)$, depression $\left(\mathrm{G}_{6}=3.52 \pm 0.16\right)$ and tension $\left(\mathrm{G}_{4}=3.34 \pm 0.14\right)$. 
Clinical and psychopathological structure of schizophrenia with diabetes mellitus 2 is determined by the severity of hallucinatory behavior $\left(\mathrm{P}_{3}=4.1 \pm 0.16\right)$, somatic concerns $\left(\mathrm{G}_{1}=4.16 \pm 0.12\right)$, excitement $\left(\mathrm{P}_{4}=3.8 \pm 0.14\right)$, depression $\left(\mathrm{G}_{6}=3.32 \pm 0.12\right)$, poor impulse control $\left(\mathrm{G}_{14}=2.8 \pm 0.12\right)$, poor attention $\left(\mathrm{G}_{11}=2.74 \pm 0.12\right)$.

The specifics of clinical and psychopathological manifestations of schizophrenia, combined with obesity, include a significant predominance of negative symptoms over positive ( $\mathrm{PN}=-4.1 \pm 0.18)$, passive/apathetic social withdrawal $\left(\mathrm{N}_{4}=3.46 \pm 0.14\right)$, poor rapport $\left(\mathrm{N}_{3}=3.54 \pm 0.12\right)$ and the presence of depressive feelings $\left(\mathrm{G}_{6}=3.65 \pm 0.16\right)$.

According to the frequency analysis of clinical-biological and social factors of formation, as well as features of clinical-psychopathological structure of schizophrenia in patients of the examined groups, diagnostic clinical-psychopathological signs (risk markers) of combination of schizophrenia with specific variants of somatic disorders are established.

The most informative signs of the risk of CVD in schizophrenia are: duration of psychotic disorder over 15 years $(\mathrm{DC}=-2.55$; $\mathrm{MI}=0.31)$; presence of depression $(\mathrm{G} 6 \geq 4)$ of moderate and higher severity $(\mathrm{DC}=-2.39 ; \mathrm{MI}=0.26)$; anxiety $\left(\mathrm{G}_{2} \geq 5\right)$ higher than moderate $(\mathrm{DC}=-2.60$; $\mathrm{MI}=0.23)$; somatic concerns $\left(\mathrm{G}_{1} \geq 4\right)$ of moderate and higher degree ( $\left.\mathrm{DC}=-1.7 ; \mathrm{MI}=0.19\right)$; history of psychoactive substance abuse $(\mathrm{DC}=-2.5 ; \mathrm{MI}=0.17)$ and emotional withdrawal $\left(\mathrm{N}_{2}>3\right)$ is higher than the weak level of severity $(\mathrm{DC}=-2.08 ; \mathrm{MI}=0.17)$.

A significant level of differentiation of the absence of CVD in schizophrenia can be achieved in the combined presence of only the first four signs: the patient's age up to 30 years ( $D C=5.23 ; M I=0.37)$; absence or minimal severity of signs of depression $\left(\mathrm{G}_{6} \leq 2 ; \mathrm{DC}=3.98\right.$; $\mathrm{MI}=0.37)$; the number of hospitalizations is not more than one per year $(\mathrm{DC}=2.52 ; \mathrm{MI}=0.28)$; absence or minimal severity of signs of anxiety $\left(\mathrm{G}_{6} \leq 2\right)(\mathrm{DC}=3.31 ; \mathrm{MI}=0.26)$. The presence of other signs may also increase the level of differentiation of CVD anti-risk to $\mathrm{p} \leq 0.01$.

Signs of the presence (risk) of type 2 diabetes in patients with schizophrenia are: lack of employment $(\mathrm{DC}=-3.28$; $\mathrm{MI}=0.52)$; presence of symptoms of excitement $\left(\mathrm{P}_{4} \geq 4\right)$ of moderate and higher degree $(\mathrm{DC}=-3.13$; $\mathrm{MI}=0.36)$; duration of psychotic disorder over 15 years $(\mathrm{DC}=-2.61$; $\mathrm{MI}=0.32)$; poor attention $\left(\mathrm{G}_{11} \geq 4\right)$ of moderate and higher severity $(\mathrm{DC}=-3.77 ; \mathrm{MI}=0.31)$.

To differentiate the anti-risk of diabetes mellitus 2 in patients with schizophrenia requires a combination of at least the first six signs: occupational employment $(\mathrm{DC}=2.39$; $\mathrm{MI}=0.33)$; absence or minimal severity of signs of depressive symptoms $\left(\mathrm{G}_{6} \leq 2\right)(\mathrm{DC}=4.01 ; \mathrm{MI}=0.29)$; 
duration of psychotic disorder up to 15 years $(\mathrm{DC}=2.13$; $\mathrm{MI}=0.29)$; the number of hospitalizations is more than one per year $(\mathrm{DC}=2.43$; $\mathrm{MI}=0.26)$; others for continuous types of schizophrenia $(\mathrm{DC}=1.64 ; \mathrm{MI}=0.17)$.

The most informative signs of the risk of obesity in schizophrenia are: lack of professional employment ( $\mathrm{DC}=-3.07 ; \mathrm{MI}=0.44)$; social isolation $(\mathrm{DC}=-6.00 ; \mathrm{MI}=0.36)$; the presence of passive-apathetic social withdrawal $\left(\mathrm{N}_{4} \geq 4\right)$ of moderate and higher severity.

The most informative signs of absence (anti-risk) of obesity in schizophrenia are: being married (official or public) $(\mathrm{DK}=6.65 ; \mathrm{MI}=1.09)$; the presence of social ties with friends and acquaintances $(\mathrm{DC}=4.22 ; \mathrm{MI}=0.71)$ and the absence or minimal severity of signs of depression $\left(\mathrm{G}_{6} \leq 2\right)(\mathrm{DC}=3.06 ; \mathrm{MI}=0.36)$.

\section{Conclusions}

The obtained results show that the combination of schizophrenia with somatic disorders is reflected in the deepening and peculiarities of psychopathological symptoms, reducing the level of social realization of patients and requires the attention of clinicians in developing treatment strategies.

\section{References}

1. The problem of multiple somatic and/or mental pathology / A.S. Avedisova, M.O. Zhabin, R.G. Akzhigitov, A.A. Gudkova // Journal of Neurology and Psychiatry. S.S. Korsakov. - 2018. - Issue. 118 (5). - S. 5-13.

2. Action plan for the prevention and control of noncommunicable diseases in the WHO European Region. Copenhagen: WHO Regional Office for Europe; - 2016.

3. Voloshyn P.V., Maruta N.O. The strategy of protecting the mental health of the population of Ukraine: happy opportunities and pereshkodi / P.V. Voloshyn, N.O. Maruta // Ukrainian Newsletter of Psychoneurology. - 2015. - Volume 23, vip. 1. -S. 5-11.

4. Global Epidemiology and Burden of Schizophrenia: Findings From the Global Burden of Disease Study / Charlson F.J. [et al.] //Schizophrenia Bulletin. - 2016. - Vol. 44 (6). - P. 1195-1203.

5. Lyubov E.B. Socio-economic burden of schizophrenia / E.B. Lyubov // Social and Clinical Psychiatry. - 2012. - Issue. 22. - No. 2. - S. 100-108.

6. Prevalence of Combined Somatic and Mental Health Multimorbidity: Patterns by Age, Sex, and Race / Ethnicity / Bobo W.V., Yawn B.P., Sauver J.L. St. [et al.] // The Journals of 
Gerontology Series A: Biological Sciences and Medical Sciences. - 2016. -Vol. 11 (71). - P. 1483-1491.

7. GBD 2017 Disease and Injury Incidence and Prevalence Collaborators. Global, regional, and national incidence, prevalence, and years lived with disability for 354 diseases and injuries for 195 countries and territories, 1990-2017: a systematic analysis for the Global Burden of Disease Study 2017. The Lancet; 2018

8. Psychosis and schizophrenia in adults: prevention and management. Clinical guideline [CG178] // London: National Institute for Health and Care Excellence. - 2014.

9. Schizophrenia is associated with excess multiple physical-health comorbidities but low levels of recorded cardiovascular disease in primary care: cross-sectional study / D.J. Smith, J. Langan, G. McLean, B. Guthrie, S.W. Mercer // BMJ Open. - 2015. - Vol. 3. - P. 808-815.

10. Gerasim'yuk I.G. Reception of schizophrenia and somatic pathology / I.G. Gerasim'yuk // World Science. - 2018. - No. 6 (34). - Vol. 5. - P.47-49.

11. Petri A. Visual medical statistics: textbook. manual / A. Petri, K. Sabin; per. from English ed. V.P. Leonov // 3rd ed., Revised. and add. - M .: GEOTAR-Media. - 2015. - 216 p.

12. Psychosomatic disorders in clinical practice / A.B. Smulevich. - M .: MEDpressinform, 2016. - $774 \mathrm{p}$. 